\title{
PERFORMANCE OF SOME GRAIN SORGHUM (Sorghum bicolor I. Moench) GENOTYPES UNDER DIFFERENT SOWING DATES IN EGYPT
}

Abd El-Raouf, M. S. A.; El-M. A. El - Metwally and A. A. Bahar Elddin Agronomy Dep., Fac. Agric., Cairo Univ., Giza, Egypt

\section{ABSTRACT}

Two field experiments were conducted in 2010 and 2011 seasons to investigate the influence of sowing date (April 7, April 27, May 17, June 6, and 26) on growth and yields of some sorghum genotypes (Shandaweel-1 hybrid, Shandaweel305 hybrid, Shandaweel-306 hybrid and Dorado cultivar) grown in clay loamy soil at Giza Governorate, Egypt. Results obtained showed that growth and yield attributes of grain sorghum were significantly affected by sowing date and genotypes in both seasons.

Delaying sowing from April to late May or June resulted in increasing growth attributes (plant height, number of green leaves, leaf area and total dry weight/plant). Late sowing in June accelerated plant maturity. Sandaweel-305 hybrid plants were the tallest and the earliest in maturity compared to the other genotypes. Sowing Shandaweel-306 hybrid at June produced the highest yield and yield components compared to the other genotypes planted at earlier sowings (April and May). The results also showed that grain yield was positively correlated with panicle weight $(r=$ $0.52)$, kernel weight panicle ${ }^{-1}(r=0.43)$, leaf area $(r=0.50)$ leaf area index $(r=53)$ and total dry weight $(r=0.43)$ suggesting that these parameters could be used to estimate sorghum grain yield.

\section{INTRODUCTION}

Grain sorghum (Sorghum bicolor L. Moench) is one of the most important cereal crops. It is one of the main stable for the world poorest and more food-insecure people. It ranks the fourth of the world cereal crops after wheat, rice and maize. Sorghum is one of the most adapted summer grain crops to drought and heat. In Egypt, Sorghum is grown in Upper Egypt from Giza to Aswan but most of the area (89 thousand hectare) is concentrated in Assiut and Sohag governorates and about 37 thousand hectare in Fayoum governorate (Ezzat et al., 2010). In addition, it is a double purpose crop; the vegetative parts are used for animal feeding in summer season where green forage crops are not quite available. The total production of grain sorghum in Egypt is less than the needs of the local consumption (Abdel-Motagally, 2010).

Grain sorghum growth, development, and yield depend on environmental conditions such as temperature and precipitation. The extent of the effect of these environmental conditions may vary depending on sowing dates and locations. Sowing date influences on grain sorghum crop through temperature and soil available water at grain germination (Vanderlip, 1993), vegetative and reproductive success (Prasad et al., 2008 and Bandiougou, 2012), number of days from sowing to physiological maturity 
and hence, yield and yield components (Jones and Johnson, 1991 and Conley and Wiebold, 2003). Alassane (2012) reported that effect of stress due to environmental factors on final yield may depend upon the growth stage in which it occurs and the genotype. Poornima et al. (2008) found that among the different dates of sowing, June $8^{\text {th }}$ sowing led to extensively higher grain yield of sweet sorghum over other dates of sowing. Increase in yield under June $8^{\text {th }}$ sowing might be due to the favorable environment prevailed during the crop growing season.

The effect of sowing date on sorghum yield changed with locations based on environmental conditions prevailing during the crop growing period. Omer (2005) found that grain sorghum and dry matter increased with July and August sowing dates, compared with June, May and April sowing dates at Toshka, Agric. Exp. Station. However, other workers (Johnson et al., 1984, Bryant et al., 1986, Francis et al., 1986, Conley and Wiebold, 2003 and Assefa and Staggenborg, 2010) found higher yields with early sowing.

Significant yield advantage of late sowings over early sowings was observed at Chillicothe, Texas, while the opposite trend was observed at Monday, Texas, where early sowings yielding more than late sowings in each of the three years of the experiment (Clark, 1997). Delaying sowing to early June was advantageous in northeast Nebraska. Irrigated grain sorghum had higher yield with late June sowing compared with early July sowing (Moomaw and Mader, 1991). Further tests were required to enable ranking of sowing date in a manner that would reflect it is affect in establishment, development and yield of grain sorghum under varied seasons and locations. Therefore, efforts are focused on increasing productivity of this crop by growing high yielding genotype under most favorable cultural conditions.

The objective of this research was to study the performance of some grain sorghum genotypes under different sowing dates at Giza, Egypt to optimize sorghum production.

\section{MATERIALS AND METHODS}

Two field experiments were conducted in 2010 and 2012 growing seasons under irrigated conditions at Agricultural experimental and Research station, Faculty of Agriculture, Cairo University, Giza governorate, Egypt to evaluate performance of three grain sorghum hybrids and one cultivar under four sowing dates in clay loamy soil. Daily maximum and minimum temperatures, during growing season (from April to October) are presented in Table 1.

Four Egyptian grain sorghum genotypes (three hybrids, i.e. Shandaweel-1, Shandaweel-305, Shandaweel-306 and one cultivar i.e. Dorado) provided by Agric. Res. Sta. were tested under five sowing dates (April $7^{\text {th }}$, April $27^{\text {th }}$, May $17^{\text {th }}$, June $6^{\text {th }}$ and $26^{\text {th }}$ ). 
Table 1. Monthly temperatures through the period from April to November in 2010 and 2011 seasons at Giza, Egypt.

\begin{tabular}{|c|c|c|c|c|c|c|}
\hline \multirow{3}{*}{ Month } & \multicolumn{6}{|c|}{ Temperature $\left(\mathbf{C}^{\circ}\right)$ at Giza, Egypt } \\
\hline & \multicolumn{3}{|c|}{2010} & \multicolumn{3}{|c|}{2011} \\
\hline & Min & Max & Mean & Min & Max & Mean \\
\hline April & 16.0 & 29.6 & 22.8 & 18.5 & 28.4 & 23.5 \\
\hline May & 19.2 & 33.9 & 26.5 & 18.7 & 32.8 & 25.7 \\
\hline June & 22.7 & 37.0 & 29.4 & 21.7 & 35.2 & 28.5 \\
\hline July & 23.9 & 36.3 & 30.1 & 23.5 & 37.3 & 30.4 \\
\hline August & 25.3 & 38.3 & 31.8 & 23.9 & 36.5 & 30.2 \\
\hline September & 23.4 & 35.8 & 29.6 & 22.7 & 35.2 & 28.9 \\
\hline October & 21.5 & 33.8 & 27.6 & 18.7 & 30.9 & 24.6 \\
\hline November & 17.1 & 28.6 & 22.8 & 13.3 & 24.5 & 18.9 \\
\hline
\end{tabular}

Source: Meteorological Observatory (Agric. Res. Center. Giza)

The experimental design was a randomized complete block design (RCBD) in split-plot arrangement with four replications. The tested sowing dates were assigned to the main plots and grain sorghum genotypes to the sub plots. The experimental unit was $20 \mathrm{~m}^{2}(4 \times 5 \mathrm{~m})$ with $60 \mathrm{~cm}$ row spacing. About 4-5 grains were hand planted in hills spaced $20 \mathrm{~cm}$ apart. After three weeks from sowing, the plants were thinned to two. The common field practices at the experimental farm were applied for the ordinary sorghum fields. The first irrigation was applied after 21 days from sowing and the next irrigations were applied at 14 days intervals. Nitrogen was applied after 21 days from sowing at the rate of $100 \mathrm{~kg} \mathrm{~N}$ per fed. From each sub plot a sample of 10 plants were randomly taken at physiological maturity from two internal rows to measure plant height, Leaf area $\left(\mathrm{m}^{2}\right)$, Leaf Area Index (LAI), Dry matter production, number of green leaves and panicle dry weight. Ten harvested panicles in each experimental unit were hand threshed and weighted. The kernel weight per panicle was calculated as an average of 10 panicle kernels weight. At maturity stage (at harvest), all plants of the other two internal rows $\left(6 \mathrm{~m}^{2}\right)$ were hand harvested and dried for estimating the biological and grain yields. Grain yield was calculated as total grain produced per fed corrected to 14 percent moisture. The Growing Degree Days was calculated using the following equation:

GDD $=\Sigma\left(\left[T_{\max }+T_{\min }\right] / 2\right)-T_{b}$

Where $T_{\max }$ and $T_{\min }$ represents maximum and minimum daily air temperature respectively, and $T_{b}$ represents base temperature of sorghum $\left(10 \mathrm{C}^{\circ}\right)$. Air temperatures were measured at nearby weather stations (Giza station).

Analysis of variance of RCBD as outlined by Gomez and Gomez (1984) was applied. Analysis of variance over years indicated significant different nearly of all treatment variables. Therefore, analysis of variance was performed separately for each year. To satisfy the assumptions of the ANOVA model, the homogeneity test of variances was verified using Bartlett's test. Mean separation test for significant effects were performed using Duncan's multiple range test (L.S.R) at $5 \%$ level of probability (Duncan, 1955). Correlation analysis was used to test the relationship between the days to $50 \%$ flowering, Plant height, Number of green leaves, leaf area, leaf 
area index, biological yield plant $^{-1}$, panicle weight, kernel weight panicle, harvest index and grain yield.

\section{RESULTS AND DISCUSSION}

Yield and yield component traits: data in (Table 2 and 3) revealed that growth, yield and its attributes were significantly affected by sowing date and grain sorghum genotypes in both seasons. May and June sowing were accompanied with the highest plant height, number of green leaves, leaf area plant $^{-1}$ and leaf area index at maturity stage in both seasons.

Table 2: Effect of sowing date on some grain sorghum traits in 2010 and 2011 seasons.

\begin{tabular}{|c|c|c|c|c|c|c|}
\hline \multirow{2}{*}{ Sowing date } & \multicolumn{6}{|c|}{ Growing seasons } \\
\hline & 2010 & 2011 & 2010 & 2011 & 2010 & 2011 \\
\hline & \multicolumn{2}{|c|}{ Plant height (cm) } & \multicolumn{2}{|c|}{ Leaf area index } & \multicolumn{2}{|c|}{ Panicle weight (g) } \\
\hline $7^{\text {th }}$ April & $131.6 \mathrm{~d}$ & $125.5 \mathrm{c}$ & $6.37 \mathrm{~d}$ & $5.36 \mathrm{~d}$ & $77.2 \mathrm{~d}$ & $80.6 \mathrm{c}$ \\
\hline $27^{\text {th }}$ April & $146.0 \mathrm{c}$ & $139.4 b$ & $7.47 \mathrm{c}$ & $7.61 \mathrm{c}$ & $83.3 \mathrm{c}$ & $87.3 \mathrm{c}$ \\
\hline $17^{\text {th }}$ May & $167.7 \mathrm{a}$ & $161.4 \mathrm{a}$ & $7.64 \mathrm{bc}$ & $8.15 b$ & 85.2 bc & $87.5 \mathrm{c}$ \\
\hline $6^{\text {th }}$ June & $162.3 b$ & $157.1 \mathrm{a}$ & $7.77 \mathrm{~b}$ & $9.99 \mathrm{a}$ & 89.4 b & $97.6 \mathrm{~b}$ \\
\hline $26^{\text {TH }}$ June & $163.5 b$ & $158.9 \mathrm{a}$ & $8.38 \mathrm{a}$ & $8.34 \mathrm{~b}$ & $108.7 \mathrm{a}$ & $108.5 \mathrm{a}$ \\
\hline \multirow[t]{2}{*}{ LSD 0.05} & 3.0 & 6.5 & 0.22 & 0.25 & 5.2 & 7.0 \\
\hline & \multicolumn{2}{|c|}{ No. of green leaves } & \multicolumn{2}{|c|}{$\begin{array}{l}\text { Days to } 50 \% \\
\text { flowering }\end{array}$} & \multicolumn{2}{|c|}{$\begin{array}{c}\text { Grains panicle-1 } \\
\text { (g) }\end{array}$} \\
\hline $7^{\text {th }}$ April & $8.0 \mathrm{c}$ & $8.0 \mathrm{c}$ & $81.0 \mathrm{a}$ & $71.0 \mathrm{~b}$ & $46.7 \mathrm{c}$ & $48.9 \mathrm{~b}$ \\
\hline $27^{\text {th }}$ April & $9.0 \mathrm{~b}$ & $8.9 \mathrm{~b}$ & $83.1 \mathrm{a}$ & $72.2 \mathrm{a}$ & $49.3 \mathrm{bc}$ & $48.6 \mathrm{~b}$ \\
\hline $17^{\text {th }}$ May & $10.6 \mathrm{a}$ & $10.2 \mathrm{a}$ & $71.5 \mathrm{c}$ & $65.4 \mathrm{~d}$ & $50.0 \mathrm{bc}$ & $49.7 \mathrm{~b}$ \\
\hline $6^{\text {th }}$ June & $10.7 \mathrm{a}$ & $10.3 \mathrm{a}$ & $69.7 \mathrm{c}$ & $61.8 \mathrm{e}$ & $52.0 \mathrm{bc}$ & $55.8 \mathrm{a}$ \\
\hline $26^{\text {TH }}$ June & $10.8 \mathrm{a}$ & $10.5 \mathrm{a}$ & $78.3 \mathrm{~b}$ & $68.6 \mathrm{c}$ & $57.8 \mathrm{a}$ & $60.9 a$ \\
\hline \multirow[t]{2}{*}{ LSD 0.05} & 0.9 & 0.5 & 2.2 & 1.2 & 3.5 & 5.2 \\
\hline & \multicolumn{2}{|c|}{ Leaf area plant ${ }^{-1}\left(\mathrm{~m}^{2}\right)$} & \multicolumn{2}{|c|}{$\begin{array}{l}\text { Dry matter plant }{ }^{-1} \\
\text { (g) }\end{array}$} & \multicolumn{2}{|c|}{$\begin{array}{l}\text { Grain yield } \text { fed }^{-1} \\
(\mathrm{~kg})\end{array}$} \\
\hline $7^{\text {th }}$ April & $50.4 \mathrm{c}$ & $50.2 \mathrm{c}$ & $103.2 \mathrm{c}$ & $103.7 \mathrm{~cd}$ & 2055 b & $1829 b$ \\
\hline $27^{\text {th }}$ April & $67.4 \mathrm{~b}$ & $67.3 b$ & $94.4 \mathrm{~d}$ & $97.6 \mathrm{~d}$ & $2062 b$ & $1995 \mathrm{~b}$ \\
\hline $17^{\text {th }}$ May & $74.7 \mathrm{ab}$ & $74.3 a b$ & $103.5 c$ & $110.3 b c$ & 2092 b & $2139 b$ \\
\hline $6^{\text {th }}$ June & 79.6 a & $79.2 \mathrm{a}$ & $114.5 b$ & $116.2 \mathrm{ab}$ & $2517 \mathrm{a}$ & $2540 \mathrm{a}$ \\
\hline $26^{\text {TH }}$ June & $73.3 \mathrm{ab}$ & $73.0 \mathrm{ab}$ & $119.5 \mathrm{a}$ & $121.3 \mathrm{a}$ & $2713 a$ & $2753 a$ \\
\hline LSD 0.05 & 12.1 & 7.8 & 4.5 & 10.4 & 220 & 374 \\
\hline
\end{tabular}

${ }^{\star}$ Means in each column followed by similar letters are not significantly different at the $5 \%$ probability level.

Number of days from sowing to physiological maturity for all genotypes decreased as sowing date was delayed. This result agrees with result of Conley and Wiebold, (2003) and Bandiougou (2012) at Manhattan and Hutchinson who reported that earlier sowing dates tended to expand the total number of days from sowing to physiological maturity. The decreased in total number of days from sowing to physiological maturity with late sowing (temperature increased) may be attribute to Cumulative Growing Degree Days $\left(\mathrm{C}^{\circ}\right)$ to reach physiological maturity to be 1467 for short season hybrids and 1849 for long season hybrid. The time period necessary to reach that GDD is function of variation in daily maximum and minimum temperature throughout the growing season, therefore may depend on sowing date.

Sorghum grain yield showed significant differences for all sowing dates. First and late June sowings recorded significantly higher grain yield (2517, 2540, 2713 and $2753 \mathrm{~kg}$ fed-1) in comparison with April or May sowings in both seasons, respectively. The percent of increase in grain yield 
with first and late of June sowing were 18\%, 24\%, $21 \%$ and $27 \%$ over first and late of April and mid of May, respectively. These results are in agreement with those of Abd El-Moneim (2005) and Omer (2010).

Table 3: Effect of genotypes on some grain sorghum traits in 2010 and 2011 seasons.

\begin{tabular}{|c|c|c|c|c|c|c|}
\hline \multirow{2}{*}{$\begin{array}{l}\text { Sorghum } \\
\text { genotypes }\end{array}$} & \multicolumn{6}{|c|}{ Growing seasons } \\
\hline & 2010 & 2011 & 2010 & 2011 & 2010 & \\
\hline & \multicolumn{2}{|c|}{ Plant height $(\mathrm{cm})$} & \multicolumn{2}{|c|}{ Leaf area index } & \multicolumn{2}{|c|}{ Panicle weight (g) } \\
\hline Shandaweel -1 & $149.0 \mathrm{c}$ & $144.5 \mathrm{c}$ & $7.6 \mathrm{~b}$ & $7.8 \mathrm{~b}$ & $95.9 \mathrm{~b}$ & $99.0 \mathrm{a}$ \\
\hline Shandaweel -305 & $180.7 \mathrm{a}$ & $171.8 \mathrm{a}$ & $6.7 \mathrm{~d}$ & $7.6 \mathrm{c}$ & $78.4 \mathrm{c}$ & $88.0 \mathrm{~b}$ \\
\hline Shandaweel -306 & $167.5 \mathrm{~b}$ & $157.5 \mathrm{~b}$ & $7.3 \mathrm{c}$ & $7.0 \mathrm{~d}$ & $102.1 \mathrm{a}$ & $97.5 \mathrm{a}$ \\
\hline Dorado & $119.6 \mathrm{~d}$ & $120.0 \mathrm{~d}$ & $8.6 \mathrm{a}$ & $9.2 \mathrm{a}$ & $78.6 \mathrm{c}$ & $84.7 \mathrm{~b}$ \\
\hline \multirow[t]{2}{*}{ LSD 0.05} & 2.9 & 4.5 & 0.3 & 0.2 & 3.9 & 4.9 \\
\hline & \multicolumn{2}{|c|}{$\begin{array}{l}\text { No. of green } \\
\text { leaves }\end{array}$} & \multicolumn{2}{|c|}{ Days to $50 \%$ flowering } & \multicolumn{2}{|c|}{$\begin{array}{c}\text { Grain weight panicle- } \\
1(\mathrm{~g})\end{array}$} \\
\hline Shandaweel -1 & 9.95 & 9.715 & $78.6 \mathrm{~b}$ & $69.2 \mathrm{~b}$ & $53.6 \mathrm{a}$ & $55.6 \mathrm{a}$ \\
\hline Shandaweel -305 & 9.97 & 9.732 & $72.6 \mathrm{~d}$ & $63.1 \mathrm{~d}$ & $50.7 \mathrm{~b}$ & $50.2 b$ \\
\hline Shandaweel-306 & 9.64 & 9.283 & $74.3 \mathrm{c}$ & $67.1 \mathrm{c}$ & $55.2 \mathrm{a}$ & $56.9 \mathrm{a}$ \\
\hline Dorado & 9.84 & 9.566 & $81.4 \mathrm{a}$ & $71.8 \mathrm{a}$ & $45.1 \mathrm{c}$ & $48.4 \mathrm{~b}$ \\
\hline \multirow[t]{2}{*}{ LSD 0.05} & NS & NS & 1.0 & 0.6 & 2.1 & 3.0 \\
\hline & \multicolumn{2}{|c|}{$\begin{array}{l}\text { Leaf area } \\
\quad\left(\mathrm{m}^{2}\right)\end{array}$} & \multicolumn{2}{|c|}{$\begin{array}{c}\text { Dry matter yield plant } \\
\text { (g) }\end{array}$} & Grain yi & $\mathrm{fed}^{-1}(\mathrm{~kg})$ \\
\hline Shandaweel -1 & $69.1 \mathrm{ab}$ & $68.8 \mathrm{~b}$ & $113.9 \mathrm{~b}$ & $114.3 \mathrm{ab}$ & $2249 d$ & $2176 \mathrm{c}$ \\
\hline Shandaweel -305 & $68.5 \mathrm{c}$ & $68.5 \mathrm{~d}$ & $97.5 \mathrm{c}$ & $108.4 \mathrm{~b}$ & $2325 \mathrm{~b}$ & $2273 b$ \\
\hline Shandaweel -306 & $68.9 \mathrm{~b}$ & $68.6 \mathrm{c}$ & $122.5 \mathrm{a}$ & $118.8 \mathrm{a}$ & 2388 a & 2359 a \\
\hline Dorado & $69.3 \mathrm{a}$ & $69.3 \mathrm{a}$ & $94.2 \mathrm{c}$ & $97.7 \mathrm{c}$ & $2263 d$ & $2266 \mathrm{~b}$ \\
\hline LSD 0.05 & 0.4 & 0.4 & 5.3 & 6.4 & 61 & 62 \\
\hline
\end{tabular}

${ }^{\star}$ Means in each column followed by similar letters are not significantly different at the $5 \%$ probability level.

Dry matter yield/ plant significantly differed because of sowing in different dates. First and late of June sowing recorded significantly higher green dry matter yield $\left(114.5,119.5,116.2\right.$ and $121.3 \mathrm{~g}$ plant $\left.{ }^{-1}\right)$ which was $14.4 \%, 19.5 \%, 12.3 \%$ and $17.2 \%$ higher over first and late of April and mid of May in both seasons, respectively. The increase in dry matter yield recorded with first and late of June sowing was as a result of increasing plant height, leaf area, total dry matter production and its partitioning in to different components (leaf, stem and ear head). These findings may be due to optimum temperature and environmental conditions at establishing and at first stage of vegetative growth period in June compared to early sowing. The present results are in conformity with those of Vanderlip (1993), Conley and Wiebold (2003), Omer (2005) and Assefa and Staggenborg (2010).

Increase in grain yield is mainly attributed to significantly higher panicle weight and kernel weight panicle ${ }^{-1}$ as well as total dry matter production, number of green leaves, leaf area, leaf area index, over first, late of April and mid of May. The results reported by Moomaw and Mader (1991) Clark (1997), Omer (2005) and Poornima et al. (2008) indicated to obtaining higher yields with late sowing. However, Johnson et al. (1984), Bryant et al. (1986), Francis et al. (1986), Conley and Wiebold (2003) and Assefa and Staggenborg (2010) found higher yields with earlier sowings. Sowing date 
Abd El-Raouf, M. S. A. et al.

influences sorghum through temperature and soil available water at grain germination and through different growth stages (Jones and Johnson, 1991 and Bandiougou, 2012).

Dorado cultivar recorded the highest value of leaf area plant ${ }^{-1}(69.3$ $\mathrm{m}^{2}$ ) and leaf area index (9.2 and 8.6) in both seasons however; plants of Shandaweel-305 hybrid were the tallest (180.7 and172cm) and the earliest (63.1 days) in maturity in both seasons.

Results of the interaction between sowing date and genotypes (Table 4 and 6) revealed that the highest leaf area index (11.71) was that of Dorado cultivar plants planted in $6^{\text {th }}$ June in second season. While, the tallest plants $(196.3$ and $192.3 \mathrm{~cm})$ and the earliest ones were those of shandawee-305 hybrid planted in $17^{\text {th }}$ May and $26^{\text {th }}$ June, respectively.

Table 4. Effect of the interaction between sowing date and genotypes on some growth traits of grain sorghum in 2010 and 2011 seasons at Giza, Egypt.

\begin{tabular}{|c|c|c|c|c|c|c|c|c|c|}
\hline \multirow[t]{2}{*}{$\begin{array}{l}\text { Sowing } \\
\text { date }\end{array}$} & \multirow[t]{2}{*}{$\begin{array}{l}\text { Grain } \\
\text { Sorghum } \\
\text { genotypes }\end{array}$} & \multicolumn{2}{|c|}{$\begin{array}{l}\text { Plant height } \\
(\mathrm{cm})\end{array}$} & \multicolumn{2}{|c|}{$\begin{array}{l}\text { Leaf area } \\
\text { index }\end{array}$} & \multicolumn{2}{|c|}{$\begin{array}{l}\text { Days to } 50 \% \\
\text { flowering }\end{array}$} & \multicolumn{2}{|c|}{$\begin{array}{l}\text { Biological yield } \\
\text { plant }^{-1} \\
(\mathrm{~g})\end{array}$} \\
\hline & & 2010 & 2011 & 2010 & 2011 & 2010 & 2011 & 2010 & 2011 \\
\hline \multirow[t]{4}{*}{ Apr.7 } & Shandaweel-1 & $123.5 \mathrm{j}$ & $121.1 \mathrm{ij}$ & $5.64 \mathrm{f}$ & 4.51 & $86.00 \mathrm{~b}$ & $72.75 \mathrm{~b}$ & $121.8 \mathrm{bc}$ & $115.4 b-d$ \\
\hline & $\begin{array}{l}\text { Shandaweel- } \\
305\end{array}$ & $154.3 e-g$ & $143.5 \mathrm{fe}$ & 6.16 ef & $5.61 \mathrm{k}$ & $79.50 \mathrm{e}$ & 67.75 ef & $74.9 \mathrm{~g}$ & $85.9 \mathrm{f}$ \\
\hline & $\begin{array}{l}\text { Shandaweel- } \\
306\end{array}$ & $140.8 \mathrm{~h}$ & $133.8 \mathrm{f}-\mathrm{h}$ & $6.45 \mathrm{e}$ & 4.451 & $73.00 \mathrm{~h}-\mathrm{j}$ & $71.00 \mathrm{c}$ & $19.3 b-d$ & $107.0 \mathrm{c}-\mathrm{e}$ \\
\hline & Dorado & $107.8 \mathrm{k}$ & $103.4 \mathrm{k}$ & $7.18 \mathrm{~d}$ & $6.84 \mathrm{hi}$ & $85.50 \mathrm{bc}$ & $72.50 \mathrm{~b}$ & $96.9 f$ & 106.6 c-e \\
\hline \multirow[t]{4}{*}{ Apr.27 } & Shandaweel-1 & $149.1 \mathrm{fg}$ & 143.5 ef & $8.28 b c$ & $6.44 \mathrm{ij}$ & $84.25 b-d$ & $74.75 \mathrm{a}$ & 113.9 c-e & $113.3 \mathrm{~b}-\mathrm{e}$ \\
\hline & $\begin{array}{l}\text { Shandaweel- } \\
305\end{array}$ & $174.2 \mathrm{~d}$ & $163.8 \mathrm{~d}$ & 5.87 ef & $8.20 \mathrm{f}$ & $75.75 \mathrm{fg}$ & $66.25 f$ & $79.0 \mathrm{~g}$ & 105.9 de \\
\hline & $\begin{array}{l}\text { Shandaweel- } \\
306\end{array}$ & $148.0 \mathrm{~g}$ & $138.4 \mathrm{fg}$ & $6.41 \mathrm{e}$ & $6.29 \mathrm{j}$ & $83.25 \mathrm{~cd}$ & $73.00 \mathrm{~b}$ & 02.8 ef & $84.5 \mathrm{f}$ \\
\hline & Dorado & $112.7 \mathrm{k}$ & $112.1 \mathrm{jk}$ & $9.30 \mathrm{a}$ & $9 \mathrm{c}$ & $89.00 \mathrm{a}$ & $74.75 \mathrm{a}$ & $82.0 \mathrm{~g}$ & $86.6 \mathrm{f}$ \\
\hline \multirow[t]{4}{*}{ May.17 } & aweel-1 & $160.9 \mathrm{e}$ & $151.2 \mathrm{e}$ & $7.32 \mathrm{~d}$ & 8.31 ef & $72.00 \mathrm{ij}$ & 67.50 ef & $113.6 \mathrm{c}-\mathrm{e}$ & $120.5 b-d$ \\
\hline & $\begin{array}{l}\text { Shandaweel- } \\
305\end{array}$ & $196.3 \mathrm{a}$ & $189.5 \mathrm{a}$ & $6.32 \mathrm{e}$ & 7.09gh & $67.25 \mathrm{k}$ & $61.00 \mathrm{~h}$ & $98.7 f$ & $109.5 \mathrm{~b}-\mathrm{e}$ \\
\hline & $\begin{array}{l}\text { Shandaweel- } \\
306\end{array}$ & $182.1 \mathrm{c}$ & $174.3 b-d$ & $7.41 \mathrm{~d}$ & $8.20 \mathrm{f}$ & $72.25 \mathrm{ij}$ & $62.25 \mathrm{gh}$ & $19.5 b-d$ & $123.0 \mathrm{bc}$ \\
\hline & Dorado & $131.5 \mathrm{i}$ & $130.4 \mathrm{~g}-\mathrm{i}$ & $9.53 \mathrm{a}$ & $9.01 \mathrm{~d}$ & $74.50 \mathrm{f}-\mathrm{i}$ & $70.75 \mathrm{c}$ & $82.4 \mathrm{~g}$ & $88.2 \mathrm{f}$ \\
\hline \multirow[t]{4}{*}{ June.6 } & aweel-1 & $156.2 \mathrm{e}$ & $153.3 \mathrm{e}$ & $8.25 b$ & $10.64 b$ & $71.00 \mathrm{j}$ & $62.50 \mathrm{~g}$ & $114.4 \mathrm{~b}-\mathrm{e}$ & $115.7 b-d$ \\
\hline & $\begin{array}{l}\text { Shandaweel- } \\
305\end{array}$ & $186.6 \mathrm{bc}$ & $178.4 b c$ & $8.54 b c$ & $8.92 \mathrm{~d}$ & $66.50 \mathrm{k}$ & $57.75 \mathrm{j}$ & $114.4 \mathrm{~b}-\mathrm{e}$ & $125.9 \mathrm{~b}$ \\
\hline & $\begin{array}{l}\text { Shandaweel- } \\
306\end{array}$ & $179.8 \mathrm{~cd}$ & $167.8 \mathrm{~cd}$ & $8.31 b c$ & $8.71 \mathrm{de}$ & $66.25 \mathrm{k}$ & $59.50 \mathrm{i}$ & $127.6 \mathrm{~b}$ & $126.2 b$ \\
\hline & Dorado & $126.4 \mathrm{ij}$ & $128.7 \mathrm{~g}-\mathrm{i}$ & $8.42 \mathrm{~b}$ & $11.71 \mathrm{a}$ & $75.00 \mathrm{f}-\mathrm{h}$ & 67.25 ef & 101.6 ef & 97.3 ef \\
\hline \multirow[t]{4}{*}{ June.26 } & Shandaweel-1 & 155.2 ef & $153.3 \mathrm{e}$ & $8.61 \mathrm{~b}$ & $9.06 \mathrm{~cd}$ & $79.50 \mathrm{e}$ & $68.50 \mathrm{de}$ & $105.90 \mathrm{~d}-$ & f106.8 c-e \\
\hline & $\begin{array}{l}\text { Shandaweel- } \\
305\end{array}$ & $192.3 a b$ & 183.6ab & $6.43 \mathrm{e}$ & $7.97 \mathrm{f}$ & $74.00 \mathrm{~g}-\mathrm{i}$ & $62.75 \mathrm{~g}$ & $120.4 \mathrm{bc}$ & $114.6 b-d$ \\
\hline & $\begin{array}{l}\text { Shandaweel- } \\
306\end{array}$ & $186.9 \mathrm{bc}$ & $173.4 b-d$ & $7.73 \mathrm{~cd}$ & $7.48 \mathrm{~g}$ & $76.75 \mathrm{f}$ & $69.75 \mathrm{~cd}$ & $143.4 \mathrm{a}$ & $153.6 \mathrm{a}$ \\
\hline & Dorado & $119.7 \mathrm{j}$ & 125.5hi & $8.31 \mathrm{bc}$ & $8.84 \mathrm{~d}$ & 82. $75 \mathrm{~d}$ & $73.50 \mathrm{ab}$ & $108.1 \mathrm{c}-\mathrm{f}$ & $110.0 \mathrm{~b}-\mathrm{e}$ \\
\hline LSD 0.05 & & 6.57 & 10.70 & 0.55 & 0.44 & 2.31 & 1.42 & 11.8 & 14.4 \\
\hline
\end{tabular}

${ }^{\star}$ Means in each column followed by similar letters are not significantly different at the $5 \%$ probability level. 
Table 5: Correlation coefficient between yield and some traits of grain sorghum crop.

\begin{tabular}{|c|c|c|c|c|c|c|c|c|c|}
\hline & 1 & 2 & 3 & 4 & 5 & 6 & 7 & 8 & 9 \\
\hline $\begin{array}{l}\text { 1-Grain } \\
\text { yield } \\
2-5 \%\end{array}$ & - & & & & & & & & \\
\hline $\begin{array}{l}\text { heading } \\
\text { 3-Plant }\end{array}$ & $-0.35^{\star \star}$ & - & & & & & & & \\
\hline height & $0.39^{* *}$ & $-0.76^{* *}$ & - & & & & & & \\
\hline 4-LAI & $0.58^{* *}$ & $-0.33^{* *}$ & $0.10 \mathrm{~S}$ & - & & & & & \\
\hline $\begin{array}{l}\text { 5-TDM } \\
\text { 6-Panicle }\end{array}$ & $0.48^{* *}$ & $-0.33^{\star *}$ & $0.46^{\star *}$ & $0.01 \mathrm{NS}$ & - & & & & \\
\hline $\begin{array}{l}\text { weight } \\
\text { 7-Kernel } \\
\text { weight/pan }\end{array}$ & $0.57^{* *}$ & $-0.20 N S$ & $0.36^{* *}$ & $0.19 N S$ & $0.75^{\star *}$ & - & & & \\
\hline 8-No. & $0.51^{* *}$ & $-0.21 \mathrm{NS}$ & $0.31^{* *}$ & $0.09 \mathrm{NS}$ & $0.73^{\star *}$ & $0.91^{* *}$ & - & & \\
\hline $\begin{array}{l}\text { Leaves } \\
\text { 9-LA }\end{array}$ & $\begin{array}{c}-0.11 \mathrm{NS} \\
0.55^{\star \star}\end{array}$ & $\begin{array}{l}-0.02 N S \\
-0.32^{\star *}\end{array}$ & $\begin{array}{l}0.06 \mathrm{NS} \\
0.18 \mathrm{NS}\end{array}$ & $\begin{array}{c}-0.03 N S \\
0.87^{\star *}\end{array}$ & $\begin{array}{l}-0.08 \mathrm{NS} \\
0.11 \mathrm{NS}\end{array}$ & $\begin{array}{c}-0.12 N S \\
0.28^{*}\end{array}$ & $\begin{array}{r}-0.11 N S \\
0.18 N S\end{array}$ & $-0.10 \mathrm{NS}$ & - \\
\hline
\end{tabular}

Table 6. Effect of the interaction between sowing date and genotypes on some grain sorghum yield traits in both seasons at.

\begin{tabular}{|c|c|c|c|c|c|c|c|}
\hline \multirow{2}{*}{$\begin{array}{l}\text { Sowing } \\
\text { dates }\end{array}$} & \multirow{2}{*}{$\begin{array}{l}\text { Grain } \\
\text { Sorghum } \\
\text { genotypes }\end{array}$} & \multicolumn{2}{|c|}{$\begin{array}{c}\text { Panicle weight } \\
\text { (g) }\end{array}$} & \multicolumn{2}{|c|}{$\begin{array}{c}\text { Grain panicle }^{-1} \\
\text { (g) }\end{array}$} & \multicolumn{2}{|c|}{$\begin{array}{c}\text { Grain yield fed }{ }^{-1} \\
(\mathrm{~kg})\end{array}$} \\
\hline & & 2010 & 2011 & 2010 & 2011 & 2010 & 2011 \\
\hline \multirow{4}{*}{ Apr.7 } & Shandaweel-1 & $91.4 \mathrm{~cd}$ & $96.6 c-e$ & $53.6 \mathrm{c}-\mathrm{g}$ & $58.5 b-d$ & $2173 \mathrm{e}$ & $1767 i$ \\
\hline & Shandaweel-305 & $55.1 \mathrm{~g}$ & $55.7 \mathrm{i}$ & $38.6 \mathrm{~m}$ & $33.5 \mathrm{i}$ & $2013 \mathrm{fg}$ & $1883 \mathrm{~g}-\mathrm{i}$ \\
\hline & Shandaweel-306 & $86.8 \mathrm{~d}$ & $93.8 \mathrm{de}$ & 52.d-h & $55.6 \mathrm{~b}-\mathrm{e}$ & $2016 \mathrm{fg}$ & $1825 \mathrm{i}$ \\
\hline & Dorado & 75.4 ef & $76.3 \mathrm{~h}$ & $42.2 \mathrm{k}-\mathrm{m}$ & $48.2 \mathrm{f}-\mathrm{h}$ & $2019 \mathrm{fg}$ & $1843 \mathrm{hi}$ \\
\hline \multirow{4}{*}{ Apr.27 } & Shandaweel-1 & $92.5 \mathrm{~cd}$ & $97.1 \mathrm{c}-\mathrm{e}$ & $48.0 \mathrm{~h}-\mathrm{j}$ & $50.9 \mathrm{e}-\mathrm{g}$ & 2064 e-g & $2040 d-f$ \\
\hline & Shandaweel-305 & $77.6 \mathrm{e}$ & 91.4 ef & $48.4 \mathrm{~g}-\mathrm{j}$ & $51.5 \mathrm{e}-\mathrm{g}$ & 2130 ef & $1966 \mathrm{f}-\mathrm{h}$ \\
\hline & Shandaweel-306 & $96.1 b-d$ & $80.3 \mathrm{gh}$ & $56.2 \mathrm{a}-\mathrm{f}$ & $46.6 \mathrm{f}-\mathrm{h}$ & 2076 e-g & $1970 \mathrm{f}-\mathrm{h}$ \\
\hline & $\begin{array}{l}\text { Dorado } \\
\text { Shandaweel-1 }\end{array}$ & $\begin{array}{c}66.9 \mathrm{f} \\
92.7 \mathrm{~cd}\end{array}$ & $\begin{array}{l}80.5 \mathrm{f}-\mathrm{h} \\
95.3 \mathrm{de}\end{array}$ & $\begin{array}{l}44.5 \mathrm{j}-\mathrm{I} \\
56.8 \mathrm{a}-\mathrm{d}\end{array}$ & $\begin{array}{l}45.5 \mathrm{gh} \\
53.1 \mathrm{~d}-\mathrm{f}\end{array}$ & $\begin{array}{c}1976 \mathrm{~g} \\
2079 \mathrm{e}-\mathrm{g}\end{array}$ & $\begin{array}{l}2006 e-g \\
2100 d-f\end{array}$ \\
\hline \multirow{2}{*}{ May.17 } & Shandaweel-305 & $77.8 \mathrm{e}$ & $80.1 \mathrm{gh}$ & $51.9 \mathrm{~d}-\mathrm{h}$ & $47.1 \mathrm{f}-\mathrm{h}$ & 2070 e-g & $2137 \mathrm{de}$ \\
\hline & $\begin{array}{l}\text { Shandaweel-306 } \\
\text { Dorado }\end{array}$ & $\begin{array}{l}97.9 \mathrm{bc} \\
72.4 \text { ef }\end{array}$ & $\begin{array}{l}98.5 \mathrm{c}-\mathrm{e} \\
76.2 \mathrm{~h}\end{array}$ & $\begin{array}{l}50.8 \mathrm{f}-\mathrm{i} \\
40.3 \mathrm{Im}\end{array}$ & $\begin{array}{c}56.4 \mathrm{~b}-\mathrm{e} \\
42.1 \mathrm{~h}\end{array}$ & $\begin{array}{c}2163 \text { e } \\
2054 \text { e-g }\end{array}$ & $\begin{array}{l}2156 d \\
2162 d\end{array}$ \\
\hline \multirow{4}{*}{ June. 6} & Shandaweel-1 & $101.0 \mathrm{bc}$ & $98.3 \mathrm{c}-\mathrm{e}$ & $51.2 \mathrm{e}-\mathrm{h}$ & $55.1 \mathrm{c}-\mathrm{e}$ & $2573 b-d$ & $2465 c$ \\
\hline & Shandaweel-305 & 71.9 e & $102.6 \mathrm{bd}$ & $54.7 \mathrm{~b}-\mathrm{f}$ & 57.4 b-e & $2443 d$ & $2583 \mathrm{c}$ \\
\hline & Shandaweel-306 & $101.1 \mathrm{bc}$ & 99.7 b-e & $56.3 \mathrm{a}-\mathrm{e}$ & $59.7 \mathrm{a}-\mathrm{d}$ & $2583 \mathrm{bc}$ & $2534 \mathrm{c}$ \\
\hline & Dorado & $77.5 \mathrm{e}$ & $90.0 \mathrm{e}-\mathrm{g}$ & $45.7 \mathrm{i}-\mathrm{k}$ & 51.0 & $2470 \mathrm{~cd}$ & $2580 \mathrm{c}$ \\
\hline \multirow{4}{*}{ June.26 } & Shandaweel-1 & $101.6 \mathrm{bc}$ & $107.6 \mathrm{ac}$ & $58.6 a-c$ & $60.2 \mathrm{a}-\mathrm{c}$ & $2660 \mathrm{ab}$ & $2503 c$ \\
\hline & Shandaweel-305 & $103.5 \mathrm{~b}$ & $110.3 \mathrm{ab}$ & $59.8 a b$ & $61.8 \mathrm{ab}$ & $2673 a b$ & $2754 b$ \\
\hline & Shandaweel-306 & $128.6 \mathrm{a}$ & $115.3 \mathrm{a}$ & $60.2 \mathrm{a}$ & $66.2 \mathrm{a}$ & $2792 a$ & $2891 a$ \\
\hline & Dorado & $100.9 \mathrm{bc}$ & 100.7 be & $52.7 d-h$ & $55.5 \mathrm{~b}-\mathrm{e}$ & $2727 a$ & $2865 a b$ \\
\hline LSD 0.05 & & 8.8 & 11.0 & 4.7 & 6.4 & 137 & 138 \\
\hline
\end{tabular}

The superiority of Dorado cultivar and Shandaweel-305 hybrid may be due to their genetic basis. Also optimum temperature and environmental conditions in late sowing might caused better establishments and growth. These results correspond with those of Prasad et al. (2008). Abd El-Moneim 
(2005) and Omer (2010) reported that plants of grain sorghum Shandaweel-6 hybrid were the tallest whereas; Dorado variety plants were the shortest ones.

Yield and yield component also showed significant differences for all genotypes (Table 3). The highest value of panicle weight plant ${ }^{-1}$, kernel weight panicle ${ }^{-1}$, total dry matter and grain yield fedan ${ }^{-1}$ were obtained from Shandaweel-306 hybrid planted in late June, it may due to the interaction between sowing date and sorghum genotypes. These results are corresponded with those of Ezzat et al. (2010).

Correlation studies (Table 4) revealed that grain yield was positive correlated with plant height $(r=0.39)$, LAl at maturity stage $(r=0.55)$, LAl $(r=$ 58) dry matter yield $(r=0.58)$, panicle weight $\operatorname{plant}^{-1}(r=0.57)$ and kernel weight panicle $(r=0.51)$.

\section{CONCLUSION}

Sowing date had consistent effect on sorghum grain yield and yield components in both seasons. Sowing in the first and late- April under relatively shorter day length when the plant exposed to colder soil weather temperatures significantly decreased growth and grain yield traits of grain sorghum. When sowing was delayed from first-April to late-June, better yields were obtained from all genotypes due to better environmental conditions and less risk of damage at early growth stages. Panicle weight and kernel weight panicle $^{-1}$ were affected by sowing date. Significant positive correlation was found between grain yield and panicle weight, kernels weight panicle-1 and total dry matter produced. All genotypes can be planted up to late May or first June in environments similar to those observed in this study. The choice of these genotypes would be preferable when sowing is delayed. Continuation of the research is necessary to confirm the results and for more investigation on the effect of sowing date on yield components for more description of increase in yield for genotypes due to delayed sowing in wider range of environment and sowing dates.

\section{REFERENCES}

Abd El-Monein Awad Allah Omer, 2005. Effect of planting date and plant population densities on the growth and yield of grain sorghum under Toshca Area. M. Sc. Thesis, Fac. Agric., Minia University, Egypt.

Abdel-Motagally (2010). Evaluation of water use efficiency under different water Regime in grain sorghum (Sorghum bicolor L. Moench). World J. Agric. Sci., 6(5): 499-505.

Alassane, M. (2012). Performance of Sorghum (Sorghum bicolor L. Moench) as an Energy Crop for Biogas Production Ph.D. Thesis, Fac. Agric. Nut. Sci., Liebig Univ., Giessen, Germany.

Assefa, Y., and Staggenborg, S.A. (2010). Grain sorghum yield with hybrid advancement and changes in agronomic practices from 1957 through 2008. Agron. J., 102:703-706. 
Bandiougou, D. (2012). Effect of planting date on growth, development and yield of grain sorghum hybrids. A thesis submitted in partial fulfillment of the requirements for degree master of science at Kansas State University Manhattan, Kansas.

Bryant, H.H.; Touchton, J.T. and Moore, D.P. (1986). Narrow rows and early planting produce top grain sorghum yields. Highlights Agric. Res. Alabama Agric. Exp. Sta., 33. p.5.

Clark, L.E. (1997). Grain sorghum production in the Texas rolling plains. Texas A\&M Univ. Agric. Res. Center at Chillicothe-Vernon. Technical Report, 97-1.

Conley, S.P. and Wiebold, W.J. (2003). Grain sorghum response to planting date. Online. Crop Management doi:10.1094/CM-2003-0204-01-RS (Accessed November, 2011).

Duncan D.B. (1955). Multiple range and Multiple "F" test. Biometric 11: 1-42.

Ezzat, E.M.; Ali, M.A. and Mahmoud (2010). Agronomic performance, genotype $\times$ environment interaction and stability analysis of grain sorghum (sorghum bicolor L. Moench). Asian J. Crop Sci., 2(4) 250 260, ISSN 1994-7879.

Francis, C. A.; Moomaw; R.S., Rajewski, J.F. and Saeed, M. (1986). Grain sorghum hybrid interactions with tillage system and planting dates. Crop Sci., 26:191-193.

Gomez, K.A. and Gomez, A.A. (1984). Statistical Procedures for Agricultural Research. Second Edition, JohnWileyand sons Interscience Publication, New York, USA.

Johnson, W. C.; Williams, E.D.; Touchton, J. T.; Eason, J.T. and Ruf, M.E. (1984). Planting dates and plant population affect grain sorghum production. Highlights Agric. Res. Alabama Agric. Exp. Sta. 31:8.

Jones, O.R. and Johnson, G.L. (1991). Row width and plant density effects on Texas high plains. Sorghum. J. Prod. Agric., 4:613-619.

Moomaw, R.S. and Mader, T.L. (1991). Double cropping grain and forage crops with small grains in the upper Midwest. J. Prod. Agric., 4:385390.

Omer, Abd El-M. A. A. (2005). Effect of planting date and plant population densities on the growth and yield of grain sorghum under Toshca Area. M. Sc. Thesis, Fac. Agric., Minia University, Egypt.

Poornima, S.; Geethalakshmi, V. and Leelamathi, M. (2008). Sowing dates and nitrogen levels on yield and juice quality of sweet sorghum. Research J. Agric. Biol. Sci. 4: 651-654.

Prasad, P.V.V.; Ristic, Z. and Staggenborg, S.A. (2008). Impact of drought and heat stress on physiologiacal development, growth and yield processes of crop plants. Advances in Agricultural Systems Modeling Series, 1:301-355.

Vanderlip, R.L. (1993). How a sorghum plant develops. Cooperative Extension Service. Kansas State University, Manhattan, K.S. 
Abd El-Raouf, M. S. A. et al.

أداء بعض التراكيب الوراثية للارة الرفيعة للحبوب تحت مواعيد زراعية مختلفة في

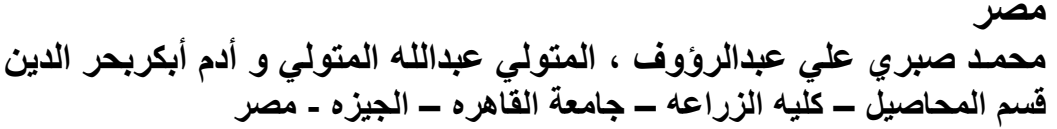

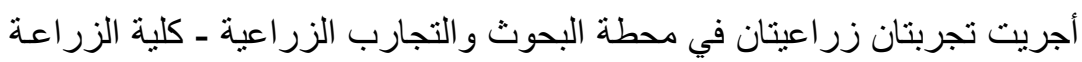

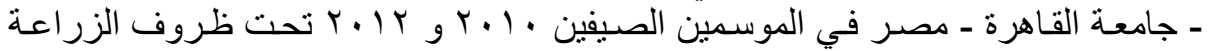

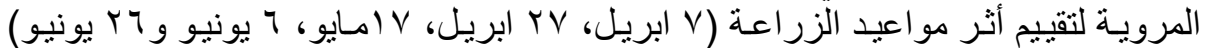

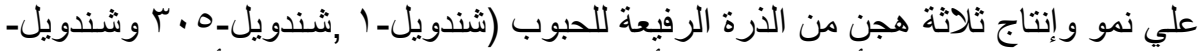

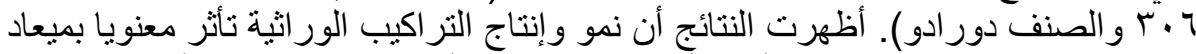

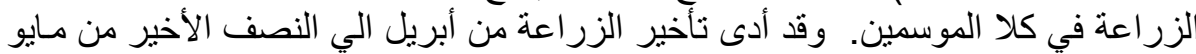

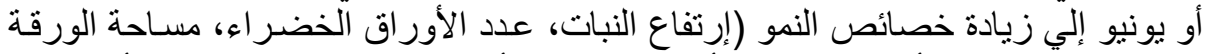

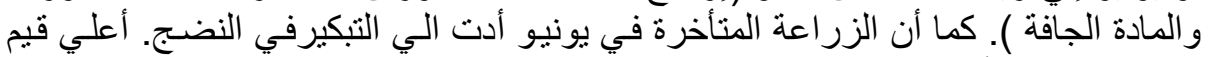

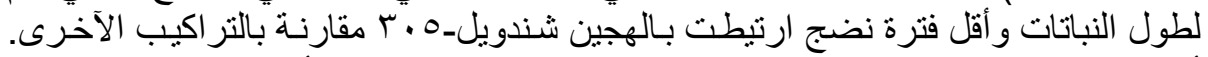

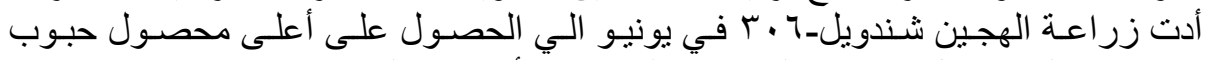

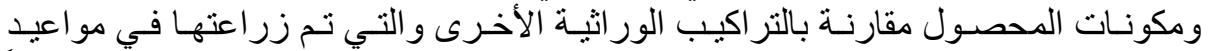

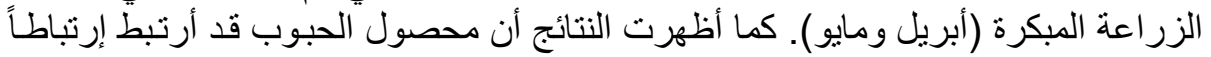

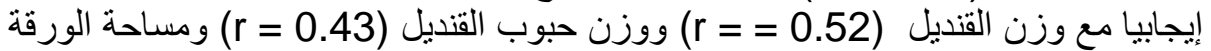

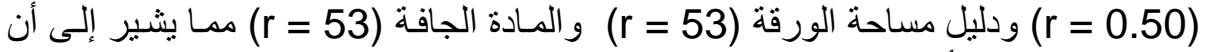
هذه المعايير يمكن أن تستخدم لتقدير محصول الحبوب لللذرة الرفيعة.

كلية الزراعة - جامعة المنصورة كلية الزراعة ـ جامعة القاهره
قام بتحكيم البحث

أ.د / الحمد نادر السيد عطيه أ.د / السيد غيث نادي الحيث 